\title{
Magnetism in Carbon: Writing Magnetic Structures with a Proton Micro-Beam on Graphite Surfaces
}

\author{
K. Schindler, D. Spemann, M. Ziese, P. Esquinazi \\ AND T. BuTZ \\ Institute for Experimental Physics II, University of Leipzig \\ Linnestr. 5, 04103 Leipzig, Germany
}

\begin{abstract}
Recent publications demonstrated the generation of magnetic order at room temperature on graphite surfaces after irradiation with protons. In this contribution we show that it is possible to produce magnetic structures in the micrometer range using a proton micro-beam by proton irradiation of the surface of highly oriented pyrolytic graphite samples. The experimental results are compared with micromagnetic simulations.
\end{abstract}

PACS numbers: 81.40.Rs, 75.50.Pp, 81.05.Uw

\section{Introduction}

At the beginning of this new millennium the rediscovery of magnetic order at room temperature in a nominally metal-free carbon-based materials [1,2] surprised as well as triggered the scepticism among physicists and material scientists. Although the formation of magnetic order in carbon was discussed theoretically in the 60's [3] and 70's [4], only a few, rather unknown experimental studies were published until the year 2000 [5]. Apart from the difficult problem to identify the contribution of magnetic impurities to the relatively weak intrinsic signal, one of the main obstacles is still the poor reproducibility of the observed magnetic order in bulk carbon. Some earlier work [6] suggested that hydrogen may play a role in triggering this order in carbon. Recently we decided to irradiate graphite samples with protons in the $\mathrm{MeV}$ energy range $[7,8]$ and showed that it is possible to produce localized (micrometer) as well as broad $(\mathrm{mm})$ regions where the magnetic order can be measured either by magnetic force microscopy (MFM) at the surface or with a SQUID magnetometer in the bulk. There are still several open questions on the origin of the magnetic order after proton irradiation in carbon samples as well as the role of hydrogen and defects. However, from the experimental point of view, we think that it is of interest to pursue highest reproducibility of the 
reported magnetic order. The main aim of this contribution is to demonstrate the possibility to magnetically write on a pure graphite surface with a proton beam as well as to show its reproducibility.

\section{Experimental details and samples}

All the results presented in this contribution were obtained from a highly oriented pyrolytic graphite sample (HOPG-ZYA, No. 29192) from Advanced Ceramics (rocking curve width $0.4^{\circ}$ ) with an impurity concentration of less than $0.5 \mu \mathrm{g}$ Fe per gram graphite as determined by particle induced X-ray emission (PIXE) spectroscopy during irradiation. The concentration of other magnetic impurities such as $\mathrm{Ni}, \mathrm{Co}$, or $\mathrm{Mn}$, was smaller than $1 \mathrm{ppm}$. The irradiation was carried out with $2.25 \mathrm{MeV}$ protons using the LIPSION accelerator on fresh HOPG surfaces - prepared by cleaving the sample just before introducing it in the vacuum chamber (within $1 \mathrm{~min}$ ) - and HOPG surfaces exposed to atmospheric conditions for long times. The results show no significant differences in the magnetic signals obtained for both surfaces indicating that the hydrogen (and water) at the graphite surface does not appear to be of mayor importance. All the MFM measurements were performed using a D3100 SPM with a NanoScope IVa controller from Digital Instruments at room temperature and operated in tapping/lift mode. Pre-magnetized low moment Si-cantilevers were used for the MFM measurements.

\section{Results}

Figure 1a shows the atomic force microscopy (AFM) image of the topography of a cross obtained with a fluence of $1 \mathrm{nC} / \mu \mathrm{m}^{2}$. The same figure shows four MFM images (b)-(e) obtained at different heights. It can be clearly seen that the magnetic signal vanishes at larger heights. Figure 2a shows MFM line scans at a given position of the crosses (white lines in Figs. 1b-e) and in Fig. 2b the maximum phase shift obtained from these scans is plotted as a function of the tip height. The data were fitted to the point probe approximation using the monopole as well as the dipole expressions, similarly as in Refs. [9, 10]. In Fig. $2 \mathrm{~b}$ one recognizes that the theoretical curves are in rough agreement with the data. Figure 3 shows the magnetic images of a cross measured at three different waiting times after irradiation. The magnetic image at the surrounding of the cross depends on time within a scale of weeks. The main magnetic image at the cross remains rather stable within this period of time. The reason for the time dependence of a magnetic signal at room temperature around the irradiated area appears to be related to the diffusion of hydrogen, which is also affected by the annealing of defects in the sample. After annealing the cross at temperatures above $800^{\circ} \mathrm{C}$ for a few hours in vacuum, the magnetic signals practically vanish [11].

Figure 4a shows the magnetic images of the same cross as in Fig. 3 and after applying a magnetic field of $3.0 \mathrm{kOe}$ in a direction along the surface normal (b) and in the opposite direction (c). Figure $4 \mathrm{~d}$ shows a line scan obtained at the 


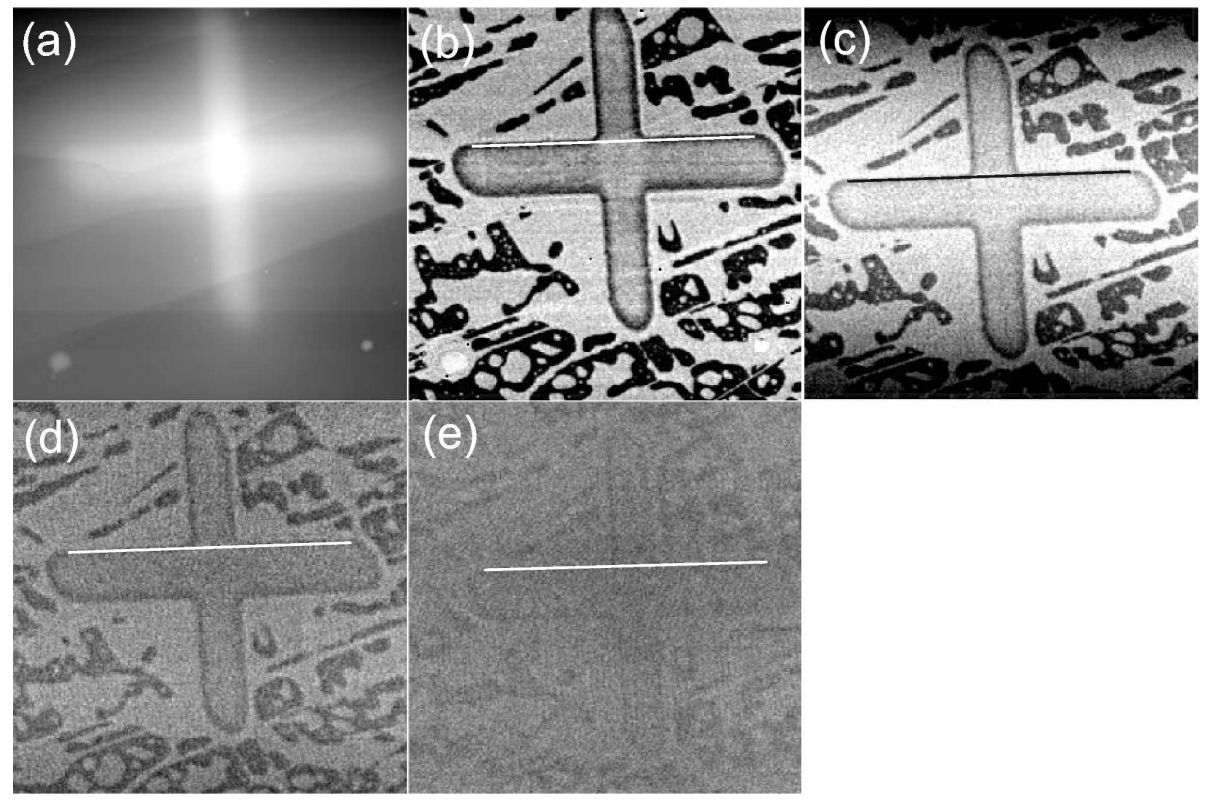

Fig. 1. Tip-height dependence of a cross produced with a proton fluence of $1 \mathrm{nC} / \mu \mathrm{m}^{2}$. (a) AFM image of the topology MFM images obtained at (b) $35 \mathrm{~nm}$, (c) $50 \mathrm{~nm}$, (d) $100 \mathrm{~nm}$, (e) $200 \mathrm{~nm}$ tip-surface heights. All images were taken on a $20 \times 20 \mu \mathrm{m}^{2}$ area.
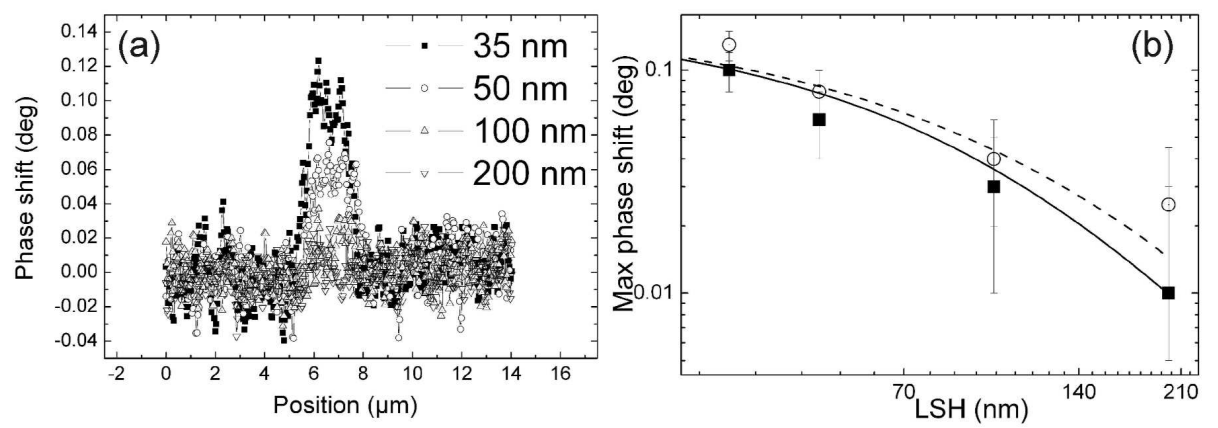

Fig. 2. (a) Line scans at the position marked by the lines in Fig. 1b-e for four different lift scan heights. (b) Maximal phase shift (taken at the left and right edges $(\mathbf{m}, \circ)$ of the cross arm) vs. lift scan height (LSH). The solid lines show the curves obtained from the point probe approximation assuming the tip being a magnetic dipole (black line) and a magnetic monopole (dashed line), respectively. The lines were computed with the parameters: effective magnetic thickness $t=1 \mu \mathrm{m}$, position of the point dipole within the tip with respect to the tip apex $\delta=100 \mathrm{~nm}$, and the effective magnetic radius $R=100 \mathrm{~nm}$. 


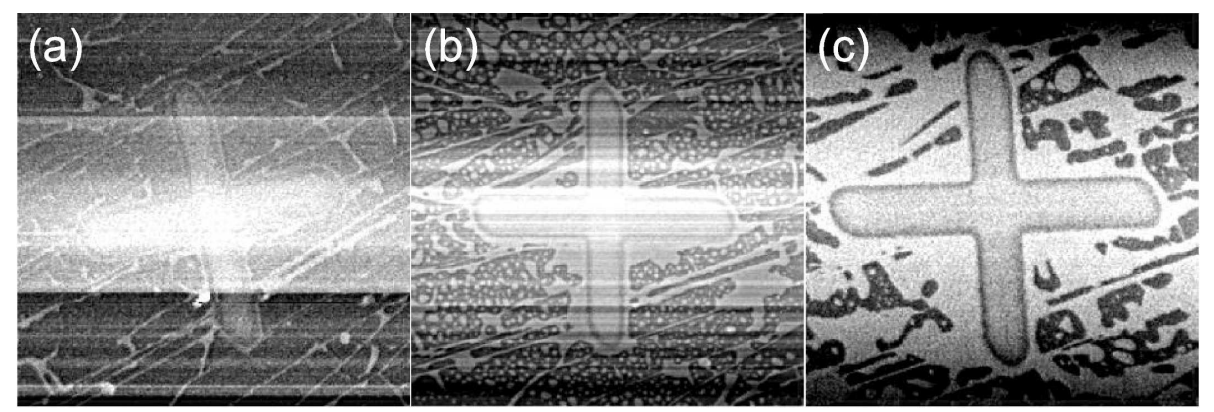

Fig. 3. Magnetic field gradient images obtained from a cross produced with a fluence of $1 \mathrm{nC} / \mu \mathrm{m}^{2}$ at three different times: (a) one day after irradiation, (b) 8 days later and (c) 34 days after irradiation. Let us note the changes observed at the surroundings of the cross. All images were taken on a $20 \times 20 \mu \mathrm{m}^{2}$ area.

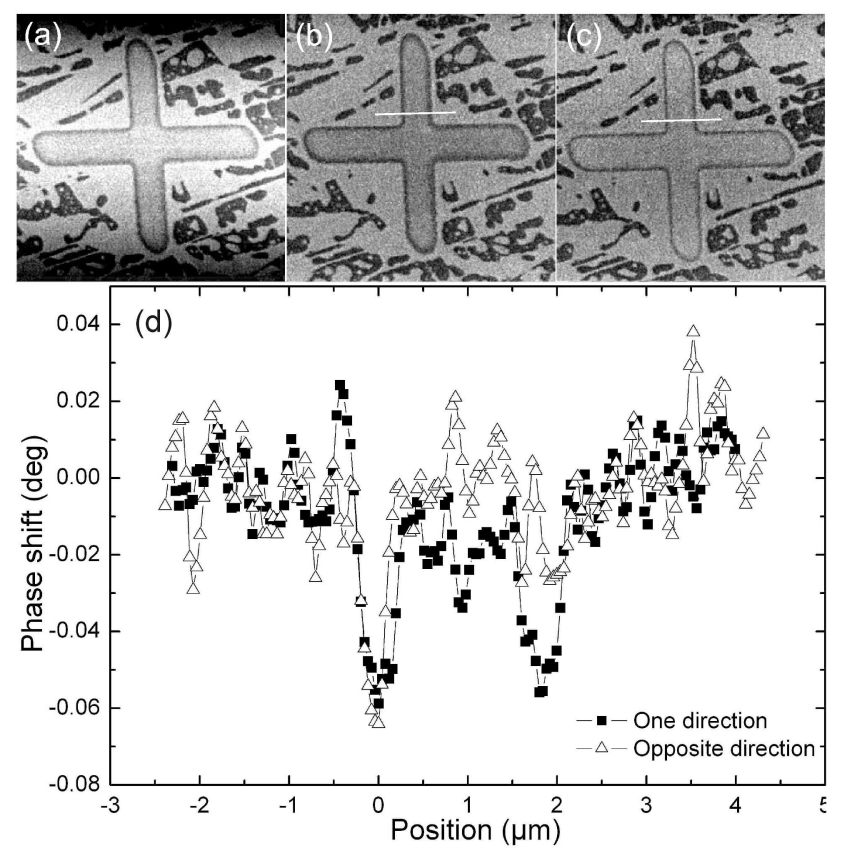

Fig. 4. (a) Magnetic image of the same cross as in Fig. 3 obtained before the application of any magnetic field. (b) The same cross after the application of a field of $3 \mathrm{kOe}$ along the surface normal and (c) in the opposite direction. (d) Line scans obtained at the positions of the white lines in parts (b) and (c).

white line position in Figs. 4b and c. There is a slight difference in the magnitude of the signal at the cross but the main magnetic domain is not affected strongly after the application of a field of this magnitude and after leaving it in remanent state. 

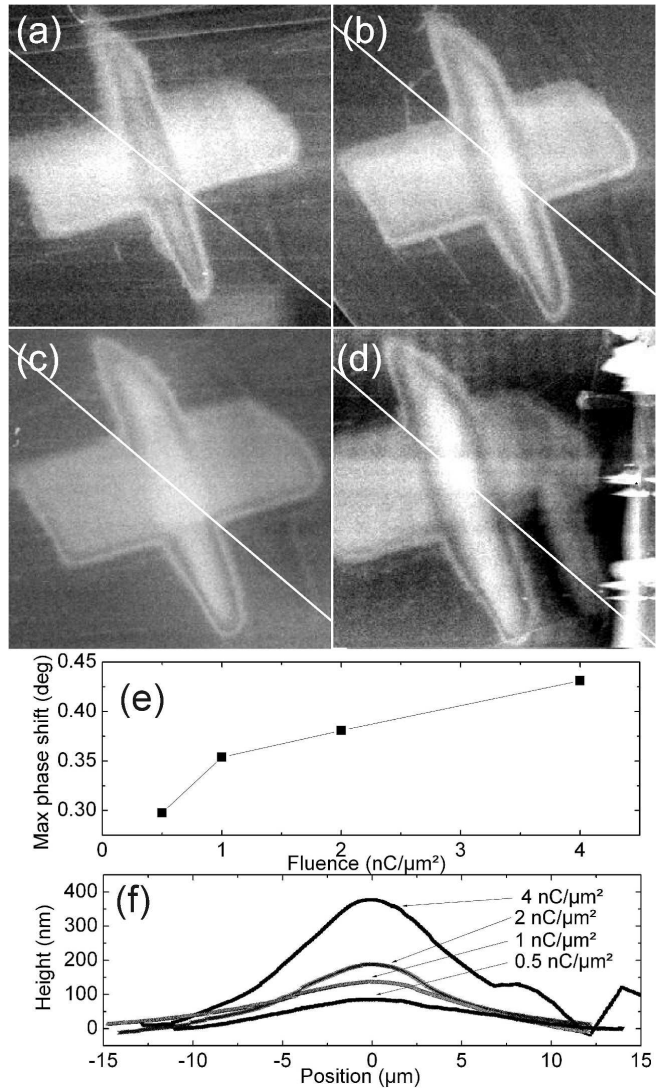

Fig. 5. Magnetic images of the "flying bird" created with a current of $120 \mathrm{pA}$ and fluences of (a) $0.5 \mathrm{nC} / \mu \mathrm{m}^{2}$, (b) $1 \mathrm{nC} / \mu \mathrm{m}^{2}$, (c) $2 \mathrm{nC} / \mu \mathrm{m}^{2}$ and (d) $4 \mathrm{nC} / \mu \mathrm{m}^{2}$. (e) Maximal phase shift as a function of fluence from the images at the white lines (a)-(d). (f) AFM line scans as a function of position of the images at different fluences.

In a preliminary study with broader crosses [11] we have observed a clear magnetic domain distribution (three domains) inside the cross. In order to study the possible geometry dependence for a multi-domain appearance, we have irradiated the graphite surface producing a broader structure. Figure 5 shows the magnetic images of what we call a "flying bird" prepared at different fluences. The observed domain patterns (a)-(d) resemble the ones obtained in broader symmetrical crosses [11]. Figure 5e shows the maximum phase shift as a function of the fluence obtained from Figs. 5a-d and (f) the AFM line scan height as a function of position.

\section{Micromagnetic simulations}

Micromagnetic simulations were carried out using the "Object Oriented Micromagnetic Framework" (OOMF) [12]. The outline of the irradiated area was 
digitized from the MFM scans to produce masks for input into the micromagnetic solver. The sample was considered to be thin and only a two-dimensional solver was used. For comparison between MFM images and the simulations we adopt the point probe approximation with a magnetic dipole moment representing the tip. In this case the measured phase shift $\Delta \phi$ is proportional to the second derivative of the vertical stray field component. From the array of magnetic dipoles obtained from the simulations the stray field and the second derivative of the $z$-component at a certain distance above the sample were calculated by adding over the dipole fields and their second derivatives. It has been experimentally shown that the height of the effective magnetic moment of the tip depends on the decay length of the stray field emanating of the sample [13] and is not identical with the lift height. Here we used an effective magnetic dipole height of $200 \mathrm{~nm}$. The simulations were performed on a sample with area $20 \mu \mathrm{m} \times 20 \mu \mathrm{m}$ using a cell size of $40 \mathrm{~nm} \times 40 \mathrm{~nm} \times 200 \mathrm{~nm}$.

The saturation magnetization $M_{\mathrm{S}}$ of the irradiated HOPG has been estimated to $2200 \mathrm{~A} / \mathrm{m}[7,8]$. For the exchange stiffness a typical value of $10 \mathrm{pJ} / \mathrm{m}$ was chosen. The magnitude and the orientation of the magnetocrystalline anisotropy are unknown. The hexagonal structure of graphite would indicate a uniaxial anisotropy; this might, however, be modified to a random anisotropy by the irradiation. Accordingly we have used both, i.e. uniaxial anisotropy along the surface normal as well as anisotropy axes randomly distributed over the unit sphere. The simulations were stopped when the torque $|M \times H| / M_{\mathrm{S}}^{2}$ reached the OOMMF default stopping criterion of $10^{-5}$. In order to obtain results on the domain structure most simulations were started with a random magnetization distribution. All simulations evolved towards a state with very few domains. However, for most choices of parameters the simulations did not reach the stopping criterion, but

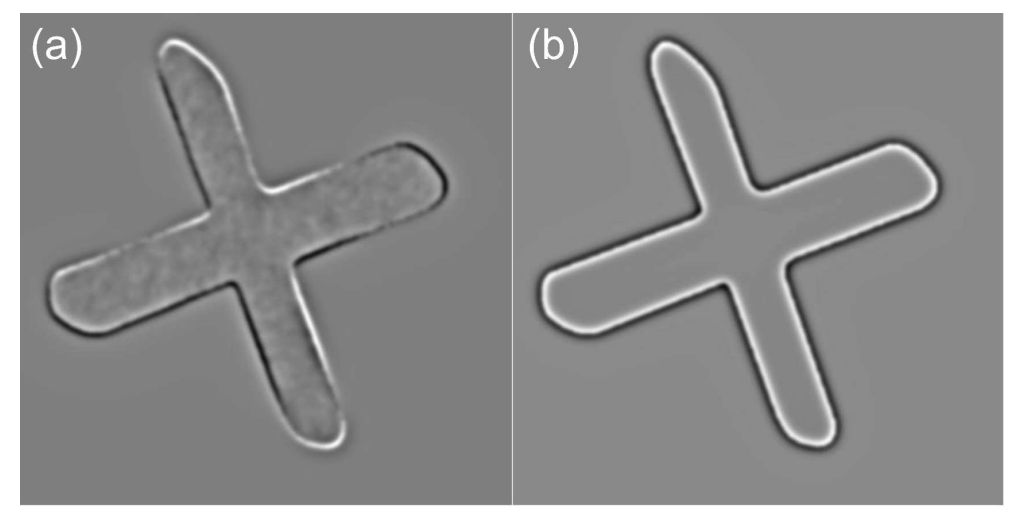

Fig. 6. Results from micromagnetic simulations. The images show the second derivative of the stray field distribution along the surface normal. Image size is $20 \mu \mathrm{m} \times 20 \mu \mathrm{m}$. The simulations were performed with a parameter set of $M_{\mathrm{S}}=2200 \mathrm{~A} / \mathrm{m}, A=10 \mathrm{pJ} / \mathrm{m}$, $K_{\mathrm{u}}=300 \mathrm{~J} / \mathrm{m}^{3}$ with (a) random and (b) uniform easy axis orientation. 
typically remained in a state with two domains with the domain wall very slowly approaching the sample boundary. The stopping criterion was reached in case of random uniaxial anisotropy with anisotropy constant $K_{\mathrm{u}}=1000$ and $300 \mathrm{~J} / \mathrm{m}^{3}$, when started from a random magnetization distribution and in case of constant uniaxial anisotropy, when started from a uniform magnetization state tilted by a small angle $<10^{\circ}$ from the surface normal.

Figure 6 shows the second derivative of the stray field for simulations with (a) random and (b) uniaxial anisotropy of magnitude $K_{\mathrm{u}}=300 \mathrm{~J} / \mathrm{m}^{3}$. In the latter case a single domain state is found, whereas the random anisotropy induces magnetic domains in the former case. Both simulations reproduce the strong stray field signal at the sample edge. The featurelessness of the MFM data observed for narrow crosses corresponds to the single domain state found in the simulations for uniform uniaxial anisotropy. Therefore we tentatively conclude that irradiated HOPG has predominantly uniaxial anisotropy with an easy axis along the $c$-axis.

\section{Acknowledgments}

This research is supported by the Deutsche Forschungsgemeinschaft under DFG ES 86/11-1 and partially by the European Union Project "Ferrocarbon" under FP6.

\section{References}

[1] Y. Kopelevich, P. Esquinazi, J.H.S. Torres, S. Moehlecke, J. Low Temp. Phys. 119, 691 (2000).

[2] P. Esquinazi, A. Setzer, R. Höhne, C. Semmelhack, Y. Kopelevich, D. Spemann, T. Butz, B. Kohlstrunk, M. Lösche, Phys. Rev. B 66, 024429 (2002).

[3] N. Mataga, Theor. Chim. Acta (Berlin) 10, 372 (1968).

[4] N. Tyutyulkov, I. Bangov, Compt. Rend. Acad. Bulg. Sci. 27, 1517 (1974).

[5] T. Makarova, Semiconductors 38, 615 (2004).

[6] K. Murata, H. Ushijima, H. Ueda, K. Kawaguchi, J. Chem. Soc., Chem. Commun., 567 (1992).

[7] K.-H. Han, D. Spemann, P. Esquinazi, R. Höhne, V. Riede, T. Butz, Adv. Mater. 15, 1719 (2003).

[8] P. Esquinazi, D. Spemann, R. Höhne, A. Setzer, K.-H. Han, T. Butz, Phys. Rev. Lett. 91, 227201 (2003).

[9] J. Lohau, S. Kirsch, A. Carl, E.F. Wassermann, Appl. Phys. Lett. 76, 3094 (2000).

[10] K.-H. Han, P. Esquinazi, J. Appl. Phys. 96, 1581 (2004).

[11] P. Esquinazi, R. Höhne, K.-H. Han, D. Spemann, A. Setzer, M. Diaconu, H. Schmidt, T. Butz, in: Carbon-Based Magnetism: an Overview of the Magnetism of Metal-Free Carbon-Based Compounds and Materials, Eds. T. Makarova, F. Palacio, Elsevier B.V. 2006, Ch. 19, p. 437.

[12] M.J. Donahue, D.G. Porter, OOMMF User's Guide, Version 1.0, Interagency Report NISTIR 6376, 1999.

[13] Th. Kebe, A. Carl, J. Appl. Phys. 95, 775 (2004). 